\title{
LoRa Indoor Coverage and Performance in an Industrial Environment: Case Study
}

\author{
Jetmir Haxhibeqiri, Abdulkadir Karaagac, Floris Van den Abeele, Wout Joseph, \\ Ingrid Moerman and Jeroen Hoebeke \\ Ghent University - imec, IDLab, Department of Information Technology \\ Email: [jetmir.haxhibeqiri,abdulkadir.karaagac,floris.vandenabeele,wout.joseph,ingrid.moerman,jeroen.hoebeke]@ugent.be \\ iGent Tower, Technologiepark-Zwijnaarde 15, B-9052 Ghent, Belgium
}

\begin{abstract}
LoRa is a long range, low power, low bit rate, single hop wireless communication technology. It is intended to be used for Internet of Things (IoT) networks, where devices are battery powered and limited bandwidth is needed. In combination with its scalability and the low end device price, LoRa is a candidate technology for low bandwidth industrial applications with a high number of communication devices spread across large areas. The use case for this paper is taken from the flower industry, where a large number of trolleys need to communicate with a server during their movement across the auction floor area. Once trolleys are outside of the auction floor they can use the public LoRaWAN network to communicate with the server, without switching communication technology. The LoRaWAN network consists of multiple end nodes and a single gateway per cell, acting as a transparent bridge between the end nodes and the network server. The measurements show that with a single LoRa gateway we can cover an indoor area of around $34000 \mathrm{~m} 2$ only with spreading factor 7 , while for spreading factor 12 the total covered area will be even higher. Also, the area outside the factory is covered when switching to spreading factor 12 . We also show that the number of nodes (trolleys) that can be served by a gateway in such a case can be as high as $\mathbf{6 0 0 0 .}$
\end{abstract}

Keywords: low power wide area networks (LPWAN), LoRa, Internet of Things (IoT),indoor industrial environment, RSSI, PER.

\section{INTRODUCTION}

In many industrial environments communication between devices and people is crucial for improving the work efficiency and automating processes. Different means of wireless communication are one of the enabling technologies in the paradigm of Industry 4.0 [1].

Low power wide area networks (LPWAN) are seen as promising technologies for those industries where the devices and tools need to be transported distant from the main factory, with requirements for low bandwidth and non-critical communication. The bit rates provided by LPWAN are up to hundreds of bits per second. However, the range is in the order of kilometers and the battery life of end devices can easily be several years. LPWANs have a single hop topology where end devices communicate directly with gateways, but not directly with each other. Gateways will act as bridges towards a central network server and the Internet. Typically end devices in an LPWAN can communicate with the gateway even under low Signal to Noise Ratio (SNR) and Received Signal Strength Indicator (RSSI) values thanks to the usage of robust modulation techniques.
Different LPWANs technologies exist such as LoRa [2], SigFox [3], RPMA [4], NB-IOT [5], Weightless [6], etc. A comparison between ultra-narrow band (UNB) and spread spectrum technologies which are currently used in LPWANs is given in [7].

The LoRaWAN technology recently gained interest from research and industrial community. The advantage of LoRaWAN is that it is an open specification on top of the LoRa physical layer, end devices are cheap and coverage is large. This makes it suitable for large-scale deployments in large industrial environments that can be covered by a single gateway only, not requiring any handover. One such industry example is the flower industry, where a lot of flower trolleys need to be monitored during their shipment to clients as well as when residing on the large auction floor. There is need for communication across long distances as well as inside the auction floor where a lot of trolleys need to be processed. Industrial environments have proven to be difficult environments in terms of wireless communication [8] [9]. However, until now, there was no study of LoRaWAN performance in such difficult environments where a lot of shielding and scattering effects occur. In this study, we will focus on the LoRaWAN performance in an industrial environment deployment consisting of a single gateway and single network server. We will evaluate the performance based on coverage (RSSI values), SNR values, packet received ratio with wrong Cyclic Redundancy Check (CRC) and packet loss ratio.

The rest of the paper is composed as follows: in Section II we will discuss related work on LoRa performance and analysis; in Section III we will give the basics of LoRa and LoRaWAN and how it works; in Section IV we will describe the setup and environment where the performance measurements were done together with our simulation model; in Section V we will give the results of measurements and simulations; Section VI will conclude the paper and will discuss potential future work.

\section{RELATED WORK}

Recently, the research community started to publish studies on different aspects of LoRa technology and LoRaWAN networks. Some of these studies are in the field of LoRaWAN coverage [10], [11], [12], [13], [14], some of them make a comparison between LoRA and other WAN technologies in 
terms of physical layer performance [15], [16], and there are a few studies on the interference mitigation for LoRa [17], [18]. To make a clarification of terms, from now onward in this paper, we will use LoRa to refer to the physical layer technology itself, whereas we will use LoRaWAN to refer to the deployment of LoRa based WANs using the LoRaWAN MAC protocol and network architecture.

Results for LoRaWAN coverage when deployed in suburban areas are reported in [10]. They reached a good coverage up to $3 \mathrm{~km}$ for spreading factor (SF) 12 and $2.3 \mathrm{~km}$ for SF 7 . The gateway was mounted at the second floor of a building and the area mainly consisted of low-rise residential buildings. In [11], authors give an introduction to LoRaWAN networks and coverage planning for such networks. They reach coverage of 2 $\mathrm{km}$ radius for SF 12 and $1.2 \mathrm{~km}$ radius for SF 7. They achieved network coverage for a city of $100 \mathrm{~km} 2$ with only 30 gateways, half the number of base stations that are used for a cellular network covering the same area. This network deployment had a density of 7000 inhabitants per gateway. Another LoRaWAN coverage study was presented in [12]. They performed measurements in an open sea environment and a city environment. Based on these measurements they achieved up to $80 \%$ packet success rate at distances lower than $5 \mathrm{~km}$ from the gateway and $60 \%$ success rate for distances from 5 to $10 \mathrm{~km}$ for the city environment. Contrary, for the open sea environment they observed up to $70 \%$ packets delivery rate at a distance of 15 $\mathrm{km}$, which is quite promising. Based on those experimental results they model the channel attenuation of LoRa [12]. Apart from outdoor coverage studies, there were two other studies for indoor LoRaWAN coverage, namely [13] and [14]. In [13], authors made excessive measurements to characterize the performance in terms of packet loss, indoor coverage, received signal strength at gateways, power consumption of end devices and delays due to duty cycle. The gateway was installed indoor and the measurements were done at the same floor, as well at the floors above and below the gateway. They report a packet loss rate of around 40\% when using SF12 at certain measurement points, which is a high percentage. However, for other SF usage the losses were under 5\%. Indoor LoRaWAN coverage in a hospital is presented in [14]. The lowest delivery ratio they achieved was $94.7 \%$ at a certain measurement point. The RSSI values were always higher than $-132 \mathrm{dBm}$, which explains the good reception ratio.

In [15], an overview of LoRa is given and a comparison with other LPWA technologies is presented. They number the advantages of using chirp modulation at the physical layer and the possibility of adopting upper layer solutions from other technologies such as IEEE 802.15.4 or 6LoWPAN. MAC and physical layers of Sigfox and LoRa are compared in [16]. LoRa uses a patented chirp spread spectrum modulation technique opposed to Sigfox that uses ultra-narrow band communication. In terms of coverage they calculated that, theoretically, LoRa can go up to $22 \mathrm{~km}$ compared to $63 \mathrm{~km}$ for SigFox. For LoRa self-interference, they calculate the cochannel interference rejection for all combinations of SFs. They claim that one transmission can be received with the same SF and in the same channel if it is received $6 \mathrm{~dB}$ higher than its interferer [16], known as the capture effect. This effect will be taken into consideration in our simulation model for assessing the scalability for our use case (see Section VI).

In [17] a self-interference study for LoRaWAN networks is presented. Two techniques covered in this study to decrease the self-interference are the usage of directional antennas and the usage of multiple base stations (gateways). They use a single base station with 600 nodes which is interfered by 4 other base stations with 600 nodes each. Using simulations, it is shown that in case of LoRaWANs usage of multiple base stations, an increase in data extraction rate of $56 \%$ was feasible compared to $32 \%$ when directional antennas were used.

In [18], authors made a study regarding the CSS modulation technique that is used in LoRa. They show that not any two CSS symbols are always orthogonal. Based on a simulation model, they show that the range is lower than ultra-narrowband solutions (like Sigfox), but that the CSS modulation technique is more robust against interference.

The network capacity is studied in [19]. They show that nodes near the gateway can send $2 \mathrm{kbit} / \mathrm{s}$ on average in uplink, while this number decreases for an increased distance from the gateway, down to only $100 \mathrm{bit} / \mathrm{s}$ on average for far away nodes. They also show the possible end devices distribution for different SFs.

\section{OVERVIEW OF LORA AND LORAWAN}

The LoRa physical layer is patented by Semtech [20] and is based on Chirp Spread Spectrum (CSS) which enables high receiver sensitivity. LoRaWAN is a MAC layer protocol and system architecture design and is standardized by the LoRa Alliance [21]. In addition to CSS, the LoRa physical layer also uses forward error correction codes to increase the robustness against noise, while the MAC layer uses an Aloha like channel access technique.

In Europe, LoRa operates in the $863-870 \mathrm{MHz}$ frequency band. It can operate in two sub-bands, one at $868 \mathrm{MHz}$ that offers three $125 \mathrm{KHz}$ LoRa channels and one at $867 \mathrm{MHz}$ having that offers five $125 \mathrm{kHz}$ LoRa channels. The gateway should be able to listen to all channels at the same time, while for end nodes it is mandatory to be able to communicate at least in the $868 \mathrm{MHz}$ sub-band. The European regulations [22] ask for adhering to a $1 \%$ duty cycle per sub-band or applying a "listen-before-talk and adaptive frequency agility" mechanism.

The narrowband signal is spread into a broadband signal by representing each bit of information with a number of chips of information. The spreading factor is related to the number of chips per bit of information. It is given as $\log 2(\mathrm{~N})$, where $\mathrm{N}$ is the number of chips per symbol. LoRa uses 6 different spreading factors ranging from 7 to 12 . The nominal bit rate is decreased by increasing the spreading factor, but the receiver sensitivity increases. The relation between data rates, spreading factor and receiver sensitivity is given in Table I. The FEC codes use a coding rate of $4 / 5$ up to $4 / 8$. The physical frame structure is composed of a preamble, an optional header and the LoRaWAN packet itself. 
TABLE I

Relations Between Data Rate, Spreading Factor and Receiver SENSITIVITY

\begin{tabular}{|c|c|c|c|}
\hline Data Rate & SF & Bit rate [kbps] & Rx sensitivity [dBm] \\
\hline \hline DR0 & 12 & 0.25 & -137 \\
DR1 & 11 & 0.44 & -135 \\
DR2 & 10 & 0.98 & -133 \\
DR3 & 9 & 1.7 & -130 \\
DR4 & 8 & 3.1 & -129 \\
DR5 & 7 & 5.4 & -124 \\
\hline
\end{tabular}

The physical frame always starts with a known chirp sequence, which helps to achieve synchronization between the transmitter and receiver. At the receiver side, the beginning of the preamble is found by correlating the received signal with the known sequence. The preamble has a variable length and can be 10 up to 65539 symbols long. According to [20], the preamble starts with a sequence of upchirps followed by two upchirps encoding the sync word. The sync word can be used to distinguish between devices from different networks. If the sync word does not match the sync word which is configured on the gateway, then the gateway will stop receiving that frame. Finally, the preamble ends with 2.25 downchirps.

The header can be implicit or explicit. The explicit header contains the payload length in bytes, the FEC code rate of the payload and the header CRC. The header is always protected with an FEC of highest code rate of 4/8. If all of these three parameters are known in advance, then it makes sense to remove the header totally, decreasing the time on air of the packet. In this case, the implicit header is applied, where the header parameters are fixed beforehand in the receiver. The payload will contain either LoRaWAN MAC layer control packets or data packets. Optionally it can be followed by the payload CRC.

The LoRaWAN MAC layer provides the medium access control mechanism that enables the communication of multiple devices with the receiver gateway. Each node transmits without sensing the channel and only adheres to the duty cycle.

LoRaWAN has a star topology architecture, where the end devices can only communicate with a LoRaWAN gateway and not directly with each other. Multiple gateways are connected to a central network server. The LoRaWAN gateways are only responsible for forwarding raw data packets from the end devices towards the server and vice versa. The network server is responsible to send the downlink packets towards end devices, if needed. The LoraWAN standard defines three classes of end devices. Class A devices support bidirectional communication by triggering the downlink communication with an uplink communication. A Class A device sends an uplink packet and after the end of the transmission it opens two downlink windows for receiving downlink traffic. The first and second receive window opens $1 \mathrm{~s}$ and $2 \mathrm{~s}$ after the end of the transmission, respectively. At any other moment, the downlink packet needs to wait for an incoming uplink transmission.

Class B devices schedule receiver slots periodically. The

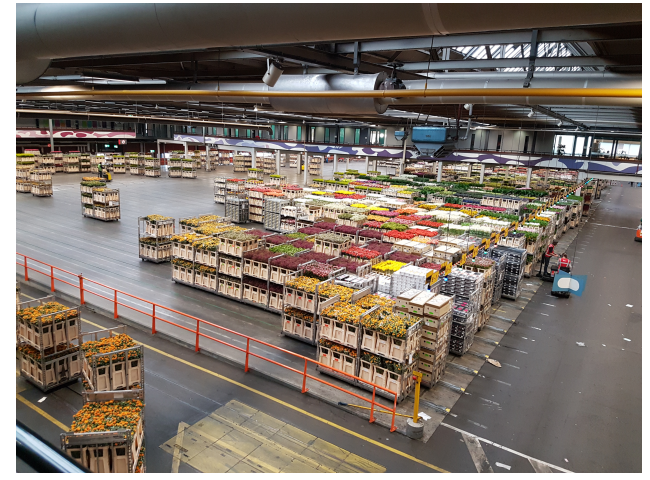

Fig. 1. The border between buffering zone and distribution zone.

period of time is determined by time synchronized beacons from the gateway. Class $\mathrm{C}$ devices can continuously receive, except when they transmit. With respect to power consumption, Class A devices are most of the time "sleeping" while Class $\mathrm{C}$ devices are all the time "awake".

The features of Class A devices are a basic set of options that every end device needs to implement in order to join a LoRaWAN network.

\section{Methods and Measurement Environment}

The chosen environment where we performed our measurements was the Royal FloraHolland Auction Center [23] located in Naaldwijk, a city near The Hague, in the Netherlands. Such a large industrial area (with $250000 \mathrm{~m} 2$ covered area and several other open areas) makes it challenging in terms of coverage when using a single technology and keeping the number of base stations as low as possible. The high number of flower trolleys (approximately 10000 going in and out every day) and their dynamicity make the environment challenging for the wave propagation, possibly having a high impact on the communication. The auction floor is composed of different areas such as: an auction hall, storing zones, buffering zones and a distribution zone. The considered measurement environment is shown in Figure 1; this is the border between the buffering zone and the distribution zone, where trolleys are processed manually by the workers. On the sides of the measurement area there are sideways (at $5.6 \mathrm{~m}$ height) where there are no trolleys. These are used as connections between the different auction floor zones.

Due to its dynamicity and due to access permissions, our measurement area was concentrated in the buffering zone (sector A) and distribution zones (sectors B and C) of the auction floor, with dimensions of 190 by 180 meters (Figure 2 ). This zone is challenging due to the high number of trolleys full of plants and flowers moving around either in parallel lines (buffer zone) or zigzag (in distribution zone). Some measurements were done outside the auction floor at the distribution gates where the tracks are filled with the flower trolleys. The LoRa gateway was installed indoor on the sideways at a height of $6 \mathrm{~m}$ above the auction floor. During the measurements, the LoRa mote was kept at a height of $1.7 \mathrm{~m}$, 
the same height as the trolleys. We used the highest transmit power allowed in the $868 \mathrm{MHz}$ frequency band, namely 14 $\mathrm{dBm}$. The measurement points and the distances between the gateway and the measurement points are shown in Figure 2.

\section{A. Measurements}

The physical layer information of the used LoRaWAN mote is provided in Table II. In order to speed up the measurement process, we made a controllable parallel measurement setup for SF 7 and SF 12. When the node which sent with SF 7 was waiting as part of its radio duty cycle we used the second node to send with SF 12. A microcontroller was used to trigger the transmission at the right timing. We chose these two spreading factors to test the highest (SF 7) and the lowest (SF 12) data rate possible, respectively $5.4 \mathrm{kbps}$ and $250 \mathrm{bps}$. In total, we obtained 33 measuring points inside the auction floor (12 points at the ground floor and 21 at the first floor on the sideways) and 13 measurement points outside the auction floor to assess the performance in the nearby vicinity of the indoor covered environment. For each measurement point we sent 50 packets for each SF.

We used the LoRANK gateway [24] which employs a WiMOD iC880A chip [25]. It is able to receive on 8 channels in parallel at sub-bands $868 \mathrm{MHZ}$ and $867 \mathrm{MHz}$ and all spreading factors. Spreading factors are orthogonal to each other so sending two packets with different SF at the same time and on the same channel will not collide. The network server saved all the received packets together with information from the physical layer: RSSI value, SNR value, CRC check, channel on which it was received and code rate. For the LoRaWAN motes we used two WiMOD iM880A [26] nodes to send packets with two different SFs one after another to speed up the measurement process. Since each LoRa mote uses counters to distinguish between consequent packets at the receiving side, we make use of this counter to detect any lost packets during the measurement. Once we moved to another location the counter was reset to 0 to make it possible to distinguish the packets from different measurement locations in the logs of the LoRaWAN server. The use case of the Royal FloraHolland Auction floor imposes several requirements to the communication. The trolleys have different update rates depending on whether they are moving or they are statically waiting at the buffer zone for the operators to pick them up. Moving trolleys should have an update rate with the server every 5 minutes, while the non-moving trolleys should have an update rate every hour. The payload packet length is 20 bytes. At any time approximately $25 \%$ of trolleys on the auction floor are moving and $75 \%$ are not moving. The requirements are to keep the packet loss rate under $10 \%$.

\section{B. Simulation Method}

In order to assess the feasibility of using a LoRaWAN network in such a large industrial environment we need to know the network scalability (the number of end nodes supported per single gateway) beforehand. For this, we prepared a simulation environment for scalability tests. The simulator is a Python
TABLE II

PHYSICAL LAYER PARAMETERS FOR MEASUREMENT SETUP

\begin{tabular}{|c|c|}
\hline Parameter & Value \\
\hline Spreading Factor & 12 and 7 \\
Channel Bandwidth & $125 \mathrm{KHz}$ \\
Code Rate & $4 / 5$ \\
Explicit Header & On \\
Channel & $868.3 \mathrm{MHz}$ \\
Payload CRC & On \\
Programmable preamble symbol length & 6 \\
Low Data rate optimization & On \\
Payload length & $15 \mathrm{bytes}$ \\
Tx power & $14 \mathrm{dBm}$ \\
\hline
\end{tabular}

script that compares the starting time of random transmissions and the transmission time length and calculates the collisions based on timing overlap and RSSI values [27]. The transmissions are done randomly from different transmissions.

In our LoRa scalability simulator we use all three channels from the sub-band of $868 \mathrm{MHz}$ and all the spreading factors. The code rate for the payload was $4 / 8$, the most robust one. We generate three vectors, the SF vector, the RSSI vector and channel vector, all with length $\mathrm{N}, \mathrm{N}$ being the number of transmitters. The distribution of different SFs can be done uniformly or with certain distribution based on the density of the nodes per area which is covered by SF. The distribution of the RSSI values is done randomly chosen from a certain range of values, or the RSSI values can be assigned based on the SF that the transmitter is using. When the SF is the highest (SF 12), then the RSSI value is assigned from a subset of values near the lowest border of the value range while for the lowest SF the RSSI value is assigned from the subset of values near the highest border of the value range. For each transmitter, a channel is assigned in a random way irrespective of the assigned SF or RSSI value. The two-dimensional matrix of packet transmission start times has a dimension of Nxn, where $\mathrm{N}$ is the number of transmitters and $\mathrm{n}$ is the number of packets that each transmitter sends (it is same for each transmitter). The matrix is populated based on the requirements of the radio duty cycle. If the transmission timings of packets from different transmitters are overlapping, and the SF and the channel is the same for both transmitters, then the packet from the transmitter received with $6 \mathrm{~dB}$ lower RSSI is counted as lost. This way we take into account the capture effect [16] which makes it possible for the packet to be received by the gateway even under collision conditions. We also calculate the number of packets received with wrong CRC. This depends on whether the collision happens during the preamble time or during the payload time of the packet. If the preamble of the packet was received uncollided, but the collision happened only during the transmission of the payload, then the packet is classified as received with wrong CRC. We calculated the total PLR for the given number of end nodes served by a gateway as a sum of the number of packets lost and the number of 

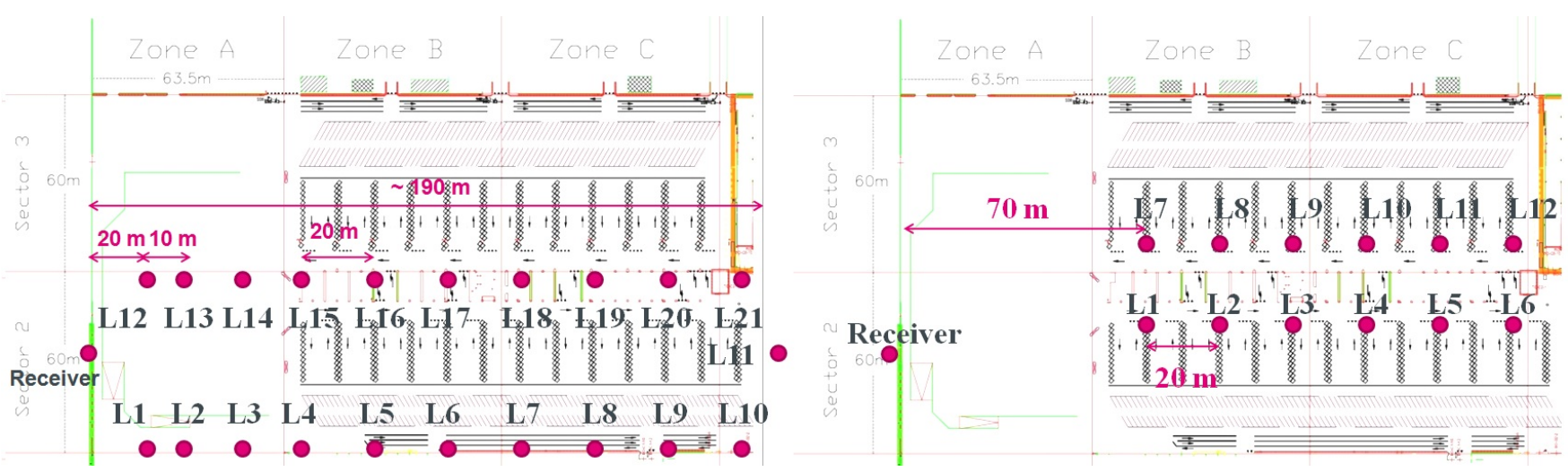

Fig. 2. Measurement points and their distance to the receiver (LoRa gateway): (left) At the sideways; (right) At the ground floor.

packets received with wrong CRC over the total number of packets sent by all nodes.

\section{RESUlts}

In a normal LPWAN the area of the zone which is covered by SF 12 is bigger than the area of the zone which is covered by SF 7 when we think of coverage zones of SFs as concentric areas around the gateway position [19]. Consequently, the number of nodes to be served with SF 12 is higher than the number of nodes to be served by any other of SFs. This makes the node distribution between different SFs nonuniform reducing thus the scalability.

In use cases like the one considered here, the main challenge is the network scalability. If the deployed LoRAWAN network can have a uniform distribution of the nodes with different SF then it can serve more nodes with a single gateway.

One of the first targets of the measurement campaign was to determine the coverage zones for the different spreading factors. However, once the zone under consideration is covered by SF 7 it can be covered by any other SF since the receiver sensitivity of any other SFs is lower than the SF 7 receiver sensitivity (see Table I). Therefore, we only performed measurements with SF 7 and SF 12.

\section{A. Measurement Results}

The RSSI values for different locations are shown in Figure 3 . The RSSI values are undoubtedly above the sensitivity level of the SF 7 which is $-123 \mathrm{dBm}$. The lowest average RSSI value was at position 12 at the ground floor which was $23 \mathrm{~dB}$ higher than the sensitivity threshold. The average values are lower for the measurement locations at the ground floor. This is due to non Line of Sight (nLOS) communication as many trolleys were blocking the LOS between the LoRa gateway and the LoRa mote. In case of measurements on the sideways, most of the time, LOS communication was ensured. Similar results were obtained using SF 12, which shows that the area under consideration can be covered by all SFs. In case of an LoRaWAN indoor network deployment, node distribution using different SFs can be done uniformly in order to increase scalability and does not have to be based on the distance from the gateway, as it should be in an outdoor LPWAN deployment. In such a case since the number of nodes using SF 12 is lower than in outdoor deployment, this will have a positive impact bit rates and will result in shorter transmission times.

The average, minimal and maximal SNR values for different locations are given in Table III and Table IV. The average SNR values $(9.5-10 \mathrm{~dB})$ were similar at different locations due to the good reception conditions. The minimal SNR values ranged from $-4 \mathrm{~dB}$ at position 11 at the ground floor to 8.8 $\mathrm{dB}$ at many other locations. The maximal values were in the interval $11-12 \mathrm{~dB}$. At the ground floor at locations 11 and 12 we had cases when the SNR value was negative. This is due to the non-LOS communication and increased number of trolleys between the LoRa mote and the gateway. Even for negative SNR values (up to $-20 \mathrm{~dB}$ ) the receiver will still be able to receive the packet due to the robustness of the modulation scheme [20].

The same measurements were done outside of the auction floor in the vicinity of the building. The measurement locations are shown in Figure 4. The gateway position is indicated by the large circle (it is installed at the same indoor position like before) while the measurement locations are indicated by smaller circles. The color of the circle of the measurement point shows the average RSSI value of the measurement point. The furthest location was around $400 \mathrm{~m}$ away from the gateway. The encircled measurement points could only be reached when using SF 12 but not when using SF 7 as the RSSI value received at the gateway was nearly at the SF 7 receiver sensitivity threshold. Also the average SNR values were under $0 \mathrm{~dB}$ at all encircled points reaching a value of $-16.4 \mathrm{~dB}$ for the furthest point from the gateway.

For the indoor measurement locations, all packets were received. However, negligible amounts of packets were received with wrong payload CRC (0.5-0.8\%). Contrary to the indoor measurement, for the outdoor measurement at the locations encircled in Figure 4, we received around 6\% of all packets with wrong payload CRC even with SF 12. For lower SF than 12 , all packets were lost. From the measurement results we can conclude that with single gateway we can cover large indoor industrial areas $(30000 \mathrm{~m} 2$ ) like the one considered in this use 

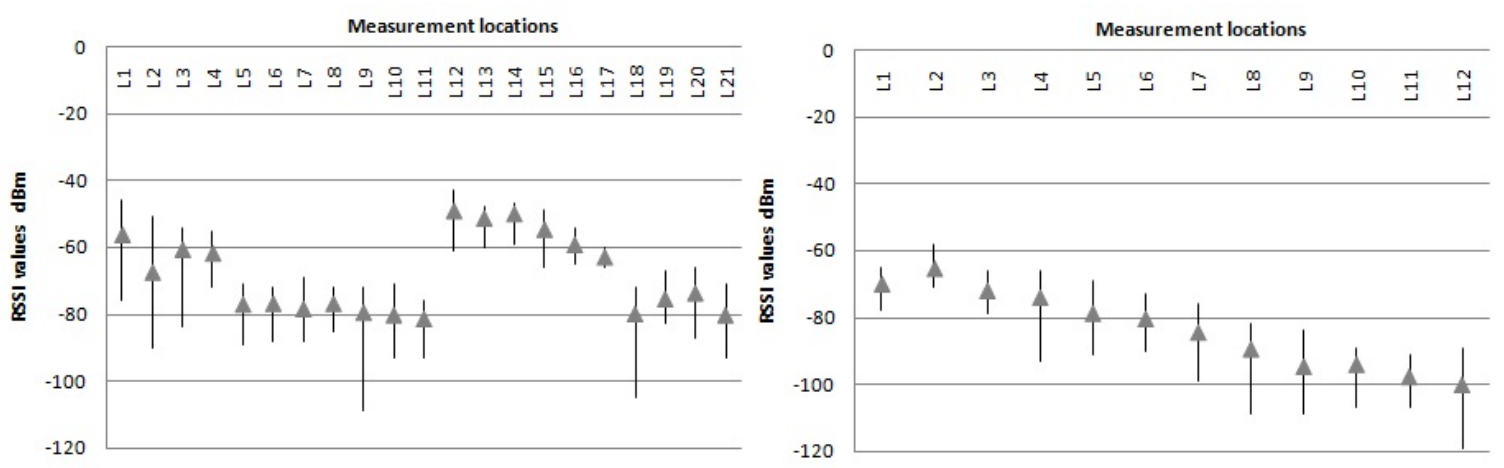

Fig. 3. RSSI values in dBm (Min, Max, and average) for each measuring location for SF7: a) At the sideways; b) At the ground floor.

TABLE III

SNR VALUES IN DB FOR THE MEASUREMENT LOCATIONS AT GROUND FLOOR

\begin{tabular}{|c|c|c|c|c|c|c|c|c|c|c|c|c|}
\hline Locations & L1 & L2 & L3 & L4 & L5 & L6 & L7 & L8 & L9 & L10 & L11 & L12 \\
\hline \hline SNR avg. $[\mathrm{dB}]$ & 9.8 & 9.7 & 9.9 & 9.7 & 9.8 & 9.9 & 9.83 & 9.98 & 9.29 & 9.65 & 8.87 & 8.65 \\
SNR min [dB] & 8.5 & 7.5 & 8.5 & 8.8 & 8.2 & 8.8 & 8.2 & 7.8 & 3 & 7 & -4 & -3.5 \\
SNR max [dB] & 11 & 11.5 & 11.2 & 11 & 11.8 & 11.8 & 11.5 & 12 & 11 & 11.5 & 11 & 11 \\
\hline
\end{tabular}

TABLE IV

SNR VALUES IN DB FOR THE MEASUREMENT LOCATIONS AT SIDEWAYS

\begin{tabular}{|c|c|c|c|c|c|c|c|c|c|c|c|}
\hline Locations & L1 & L2 & L3 & L4 & L5 & L6 & L7 & L8 & L9 & L10 & L11 \\
\hline \hline SNR avg. [dB] & 9.8 & 9.6 & 9.8 & 9.63 & 9.8 & 9.8 & 10 & 9.7 & 10 & 9.64 & 9.1 \\
SNR min [dB] & 8.5 & 7.5 & 8.8 & 8.8 & 6.5 & 8.8 & 8.5 & 6.5 & 8.8 & 7 & 0.2 \\
SNR max [dB] & 11.8 & 10.8 & 11.2 & 10.8 & 11.8 & 11.5 & 11.5 & 11.8 & 11.5 & 11 & 11 \\
\hline Locations & L12 & L13 & L14 & L15 & L16 & L17 & L18 & L19 & L20 & L21 \\
\hline SNR avg. [dB] & 9.79 & 10 & 9.85 & 9.76 & 9.86 & 9.76 & 9.72 & 9.7 & 9.71 & 9.84 \\
SNR min [dB] & 8.5 & 8.8 & 8.8 & 8.8 & 8.5 & 8.5 & 6 & 8.5 & 8.2 & 8.2 \\
SNR max [dB] & 11 & 11.2 & 11.5 & 11.5 & 12 & 11 & 11.2 & 11.8 & 11.8 & 11 \\
\hline
\end{tabular}

case with all the SFs. However, the coverage outside the indoor environment will decrease quickly and will be low due to the large attenuation of the walls. This makes it possible to design a LoRaWAN network with uniform SF node distribution rather than non-uniform distribution due to different coverage zones. We did not observe any packet loss at indoor locations. The main cause of the losses at this LoRaWAN deployment case come from network self-interference. In subsection V B we present simulation results for such a case using the LoRa simulation model described in subsection IV B.

\section{B. Simulation Results}

We calculated the time on air for a packet with a payload of 20 bytes, being $1318.91 \mathrm{~ms}$ for SF 12 and $66.82 \mathrm{~ms}$ for SF 7. So even for SF12, we can send such packets every 5 minutes as the waiting time due to the radio duty cycle requirements is only $139 \mathrm{~s}$. The other physical layer data that we took into account were: channel width of $125 \mathrm{KHz}$, use of the explicit MAC header, 8 symbols programmed preamble length, payload CRC enabled and low data rate optimization enabled.

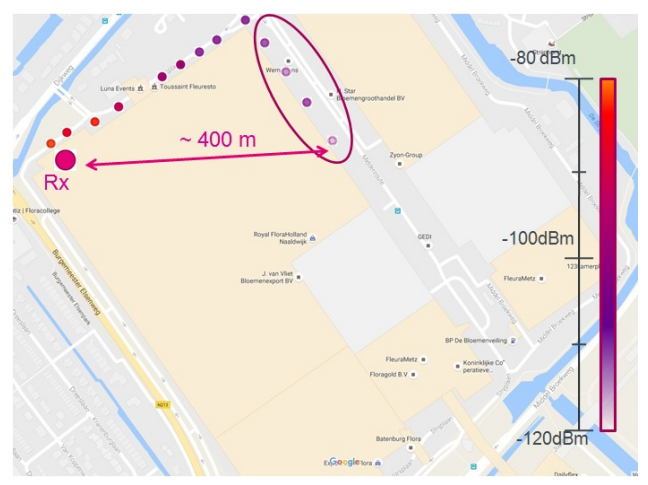

Fig. 4. The outdoor measurement locations and their RSSI values with SF 12.

For the simulation, the three channels in the $868 \mathrm{MHz}$ subband were used. We used 6 different SFs, so we had 18 logical channels in total. The SF vector was populated uniformly, while the RSSI values vector was populated randomly with values from the range $[-110,-60] \mathrm{dBm}$. These values were the RSSI range of the values we measured at factory. The matrix 


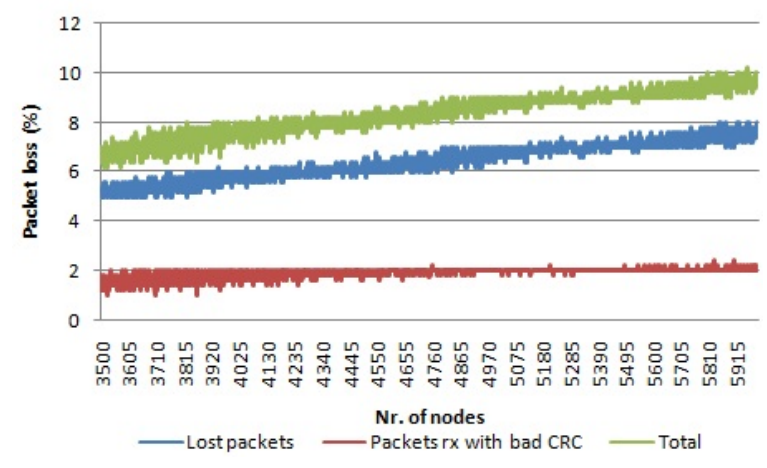

Fig. 5. Scalability simulations.

of packet transmission start time is populated based on the requirements of the trolleys update rate. This will not violate the radio duty cycle as the update rate of the trolleys is in any case lower than the radio duty cycle.

In Figure 5 the number of nodes that can be served by a single gateway resulting from this simulation is shown. Up to 6000 nodes can be served with a single gateway under the aforementioned conditions and update rates. The lost packets line shows the percentage of packets that collided on the preamble time and that were never received. The packet received with bad CRC line shows the percentage of packets that were collided on the payload time and the total line is the addition of both previous ones. For number lower than 3500 end devices per gateway the total losses will be lower than $6 \%$.

The simulation model takes into consideration only the deployment of networks with single gateway. If there will be two gateways in each other proximity then the scalability can be increased as the packets that experience collisions as seen from the first gateway can be picked up by the other gateway. Another factor impacting the scalability of the network is the interference caused by other technologies that operate in the same band. This is not considered in this case.

\section{CONCLUSION AND FUtURE WORK}

In this paper, we showed the coverage and performance results for an LPWAN indoor deployment. We deployed a LoRaWAN network with a single gateway and a single network server. The measurements to determine the coverage area and the performance were performed in a real indoor industrial environment, namely a flower auction warehouse. In most cases, the communication path between the LoRaWAN end device and the gateway was blocked by the metallic flower trolleys.

Based on the measurements we can conclude that we are able to cover the whole industrial area under consideration, with a surface of $34000 \mathrm{~m} 2$, with SF 7. In general, the SNR values were above $0 \mathrm{~dB}$ with some negative values at some measuring locations. The average RSSI values were above $-100 \mathrm{dBm}$ at all measuring locations. We did not have any packet losses except some negligible number of packets received with wrong payload CRC $(0.5-0.8 \%)$ for the indoor measuring points.

For the outdoor measuring locations, we could have communication only with SF 12 with the most distant measuring point, at $400 \mathrm{~m}$. At those points, we had up to $6 \%$ of packets received with wrong CRC. Communication using SF 7 was not possible. The average SNR values were negative with a negative peak of $-16.4 \mathrm{~dB}$.

To calculate the network scalability in such an environment we made use of a simulation model. We took into account the update rate of the end devices: $75 \%$ of the nodes sending a 20 byte packet every hour and $25 \%$ of them sending a 20 byte packet every 5 minutes. In such a network configuration, we are able to serve up to 6000 end nodes while keeping packet loss ratio below $10 \%$.

By adding a second gateway the number of end nodes that can be served can be further increased. However, this needs to be evaluated beforehand. The simulation model mentioned in this paper could be extended by taking into account the reception of packets by two gateways simultaneously, which will increase the guarantees that the packet will be received as well as the total network performance.

\section{ACKNOWLEDGMENT}

HYCOWARE is a project realized in collaboration with imec. Project partners are Egemin, Aucxis and Intation, with project supported by VLAIO (Flanders Innovation and Entrepreneurship).

\section{REFERENCES}

[1] Drath, R. and Horch, A., Industrie 4.0: Hit or Hype? IEEE Industrial Electronics Magazine, Vol. 8, 2014, pp. 56-58.

[2] https://www.lora-alliance.org/what-is-lora/technology

[3] https://www.sigfox.com/

[4] How RPMA Works, A White Paper by INGENU.

[5] Landstrom, S., et al. "NB-IoT: A sustainable technology for connecting billions of devices", Ericsson Technology Review, 22 April 2016

[6] http://www.nwave.io/

[7] "A comparision of UNB and spread spectrum wireless technologies as used in LPWA M2M applications", A white paper by Real Wireless.

[8] Haxhibeqiri, J, et al. "Wireless handover performance in industrial environments: A case study." Emerging Technologies and Factory Automation (ETFA), 2016 IEEE 21st International Conference on. IEEE, 2016. doi: 10.1109/ETFA.2016.7733578

[9] Gong, X., et al. A Measurement-Based Wireless Network Planning, Monitoring, and Reconfiguration Solution for Robust Radio Communications in Indoor Factories, IET Science, Measurement \& Technology, 2015. doi: 10.1049/iet-smt.2015.0213

[10] Augustin, A., et al. "A Study of LoRa: Long Range \& Low Power Networks for the Internet of Things." Sensors 16.9 (2016): 1466. doi:10.3390/s16091466

[11] Centenaro, M., et al. "Long-range communications in unlicensed bands: The rising stars in the IoT and smart city scenarios." arXiv preprint arXiv:1510.00620 (2015). doi: 10.1109/MWC.2016.7721743

[12] Petajajarvi, J., et al. "On the coverage of LPWANs: range evaluation and channel attenuation model for LoRa technology." ITS Telecommunications (ITST), 2015 14th International Conference on. IEEE, 2015. doi: 10.1109/ITST.2015.7377400

[13] Neumann, P., et al. "Indoor Deployment of Low-Power Wide Area Networks (LPWAN): a LoRaWAN case study.", IEEE 12th International Conference on Wireless and Mobile Computing, Networking and Communications (WiMob), New York, NY, 2016, pp. 1-8. doi: 10.1109/WiMOB.2016.7763213 
[14] Petjjrvi, J., et al. "Evaluation of LoRa LPWAN technology for remote health and wellbeing monitoring." 2016 10th International Symposium on Medical Information and Communication Technology (ISMICT). 2016. doi: 10.1109/ISMICT.2016.7498898

[15] Vangelista, L., Zanella, A. and Zorzi, M. "Long-Range IoT Technologies: The Dawn of LoRa." Future Access Enablers of Ubiquitous and Intelligent Infrastructures. Springer International Publishing, 2015.

[16] Goursaud, C. and Gorce, J.M. ”Dedicated networks for IoT: PHY/MAC state of the art and challenges." EAI endorsed transactions on Internet of Things (2015).

[17] Voigt, T., et al. "Mitigating Inter-network Interference in LoRa Networks." arXiv preprint arXiv:1611.00688 (2016).

[18] Reynders, B. and Pollin, S., "Chirp spread spectrum as a modulation technique for long range communication," 2016 Symposium on Cоттиnications and Vehicular Technologies (SCVT), Mons, 2016, pp. 1-5. doi: 10.1109/SCVT.2016.7797659

[19] Mikhaylov, K., Petaejaejaervi, J. and Haenninen, T. "Analysis of Capacity and Scalability of the LoRa Low Power Wide Area Network Technology," European Wireless 2016; 22th European Wireless Conference, Oulu, Finland, 2016, pp. 1-6.

[20] Seller, O. B. A., and Sornin, N., "Low power long range transmitter." EP 2763321A1, issued Aug 6, 2014.

[21] LoRaWAN ${ }^{T M}$ Specifications, V1.0, January 2015.

[22] ETSI, ERM TG28. "Electromagnetic compatibility and Radio spectrum Matters (ERM); Short Range Devices (SRD); Radio equipment to be used in the $25 \mathrm{MHz}$ to $1000 \mathrm{MHz}$ frequency range with power levels ranging up to $500 \mathrm{~mW}$." European harmonized standard EN 300.220: v2.

[23] Royal Flora Holland company website, Retrieved onJanuary 23, 2017, from https://www.royalfloraholland.com.

[24] Ideetron company products website, Retrieved onJanuary 23, 2017, from http://www.ideetron.nl/lora/

[25] WiMOD iC880A v0.15, Datasheet, IMST GmbH, March 3, 2015.

[26] WiMOD iM880A v0.8, Datasheet, IMST GmbH, July 27, 2013.

[27] Haxhibeqiri, J.; Van den Abeele, F.; Moerman, I.; Hoebeke, J. LoRa Scalability: A Simulation Model Based on Interference Measurements. Sensors 2017, 17, 1193. doi: 10.3390/s17061193 